\title{
SOME NEW RESULTS ON VIBRATIONAL PROPERTIES OF AMORPHOUS GROUP IV SEMICONDUCTORS
}

\author{
M. A. GRADO CAFFARO \\ Consultant, Madrid (SPAIN) \\ M. GRADO CAFFARO \\ CSIC, Madrid (SPAIN)
}

(Received April 2, 1993; in final form May 5, 1993)

For neutron scattering, an interesting formula is derived from the coherent one-phonon dynamic structure factor. In this derivation, phonon density of states is involved; this density is related to spectra due to structural disorder, which is investigated. Our considerations refer to amorphous Group IV semiconductors.

\section{INTRODUCTION}

Inelastic neutron scattering has been employed extensively in order to analyze vibrational spectra of a large number of amorphous semiconductors. In the following, we shall study one-phonon dynamic structure factor and phonon density of states. Calculations related to this density are relevant in the context of inelastic neutron scattering for amorphous $\mathrm{Ge}$ and $\mathrm{Si}$.

We shall consider a density of states corresponding to the inelastic scattering spectrum of a one-component amorphous quasi-harmonic solid. On the other hand, we can claim that reliable measurements of phonon density of states for Group IV semiconductors were performed by various workers $[1,2]$.

\section{THEORY}

Following Alben [3], the coherent one-phonon dynamic structure factor for a model structure is given by [4]:

$$
\begin{aligned}
\mathrm{S}(\overrightarrow{\mathrm{q}}, \mathrm{E})=(\hbar / \mathrm{m} \omega)\left(\mathrm{v}+\frac{1}{2}\right) \sum_{\mathrm{n}}\left\{\left|\overrightarrow{\mathrm{q}} \cdot \sum_{\alpha}\left[\overrightarrow{\mathrm{u}}_{\mathrm{n} \alpha} \exp \left(\mathrm{i} \overrightarrow{\mathrm{q}} \cdot \overrightarrow{\mathrm{r}}_{\alpha}\right)\right]\right|^{2} \delta\left(\mathrm{E}-\mathrm{E}_{\mathrm{n}}\right)\right. \\
\times \exp (-2 \mathrm{~W}) \quad(\mathrm{i}=\sqrt{-1})
\end{aligned}
$$

where $m$ is the mass, which is the same for all sites; $\hbar$ is the modified Planck constant; $\omega$ is the angular frequency; $v$ is the vibrational quantum number; $\vec{q}$ is 
transferred momentum; $\overrightarrow{\mathrm{u}}_{\mathrm{n} \alpha}$ is the normalized eigenvector corresponding to displacement of the $\alpha^{\text {th }}$ atom and referred to $n^{\text {th }}$ mode; the vectors $\vec{r}_{\alpha}$ are the equilibrium positions; $\delta$ means Dirac function; $E$ is energy; $E_{n}$ is the energy of the $n^{\text {th }}$ mode; and $\mathrm{W}$ is here the site-independent Debye-Waller factor.

Now we introduce [3]:

$S^{\prime}(\vec{q}, E)=S(\vec{q}, E) /\left[\frac{\hbar}{m \omega}\left(v+\frac{1}{2}\right) \frac{1}{3} q^{2} \exp (-2 W)\right]$

where $\mathrm{q}$ is the modulus of $\overrightarrow{\mathrm{q}}$.

From (1) and (2) it is deduced:

$\left|S^{\prime}(\overrightarrow{\mathrm{q}}, E)\right|=\mathrm{S}^{\prime}(\overrightarrow{\mathrm{q}}, \mathrm{E}) \leqslant 3 \sum_{\mathrm{n}}^{\mathrm{N}} \delta\left(\mathrm{E}-\mathrm{E}_{\mathrm{n}}\right) \sum_{\alpha}^{\mathrm{N}}\left|\overrightarrow{\mathrm{u}}_{\mathrm{n} \alpha}\right|^{2}$

On the other hand, it is well-known that $S^{\prime}(\vec{q}, E) \equiv g(E)$ in the incoherent limit of $q$ (large $q)$ [3]; $g(E)$ is the phonon density of states. Therefore we can establish:

$g(E)=3 \sum_{n}^{N} \delta\left(E-E_{n}\right) \sum_{\alpha}^{N}\left|\vec{u}_{n \alpha}\right|^{2}$

We also can write [5]:

$g(\omega)=\frac{1}{3} q^{2} N^{-1} \exp (-2 W) \sum_{n}^{N} \delta\left(\omega-\omega_{n}\right)$

By changing $E$ with $\omega$ in (4) $(\hbar=1$ in atomic units), we have:

$\int_{\Omega} g(\omega) d \omega=3 \sum_{n, \alpha}^{N}\left|\vec{u}_{n \alpha}\right|^{2}$

by taking into account $[6,7]$ :

$\int_{\Omega} \delta\left(\omega-\omega_{n}\right) \mathrm{d} \omega=1$

where $\Omega$ is the $\omega$-domain and $\omega_{n}$ is the vibrational eigenfrequency of the $n^{\text {th }}$ normal mode of the solid.

From (5) we obtain:

$\int_{\Omega} g(\omega) d \omega=\left(q^{2} / 3\right) \exp (-2 W)$

since $\sum_{n=1}^{N} 1=N$ 
Finally from expressions (6) and (7) we get:

$$
\sum_{n, \alpha}^{N}\left|\vec{u}_{n \alpha}\right|^{2}=(q / 3)^{2} \exp (-2 W)
$$

Formula (8) involves vibrational eigenvectors and refers to large q. Next we shall consider structural disorder in amorphous Group IV semiconductors; in the context of tetrahedrally coordinated amorphous semiconductors, the first-order infrared spectrum due to structural disorder can be expressed as follows $[8,9,10]$ :

$\xi_{2}^{(s)}(\omega)=\omega^{-2}|\mu(\omega)|^{2} \mathrm{~g}(\omega)$

where $\mu(\omega)$ is the dipole-moment matrix element in the frequency domain.

Now it is interesting to evaluate the important quantity $\int_{\Omega} \omega \xi_{2}^{(s)}(\omega) \mathrm{d} \omega$; we have:

$\int_{\Omega} \omega \xi_{2}^{(\mathrm{s})}(\omega) \mathrm{d} \omega=3 \sum_{\mathrm{n}}^{\mathrm{N}} \sum_{\alpha}^{\mathrm{N}}\left|\overrightarrow{\mathrm{u}}_{\mathrm{n} \alpha}\right|^{2} \int_{\Omega} \omega^{-1}|\mu(\omega)|^{2} \delta\left(\omega-\omega_{\mathrm{n}}\right) \mathrm{d} \omega$

(see eq. (4))

We get as final result (see refs. $[6,7])$ :

$\int_{\Omega} \omega \xi_{2}^{(s)}(\omega) \mathrm{d} \omega=3 \sum_{\mathrm{n}}^{\mathrm{N}} \omega_{\mathrm{n}}^{-1}\left|\mu\left(\omega_{\mathrm{n}}\right)\right|^{2} \sum_{\alpha}^{\mathrm{N}}\left|\overrightarrow{\mathrm{u}}_{\mathrm{n} \alpha}\right|^{2}$

\section{DISCUSSION}

At large q, coherent processes are not relevant. In fact, eqs. (4) and (11) refer to this situation. It is well-known that inelastic neutron scattering at fixed and large $\mathrm{q}$ makes possible the direct measurement of the phonon density of states. This density can be obtained theoretically by means of eq. (5) or by using the expression $\mathrm{g}(\omega)=3 \Sigma_{\mathrm{n}}^{\mathrm{N}} \delta\left(\omega-\omega_{\mathrm{n}}\right) \Sigma_{\alpha}^{\mathrm{N}}\left|\overrightarrow{\mathrm{u}}_{\mathrm{n} \alpha}\right|^{2}$ (see eq. (4)). In addition, eq. (8) represents a result derived from the conjunction of the previous expression and eq. (5). Equation (8) can be expressed as: $\left(\sum_{n, \alpha}^{N}\left|\vec{u}_{n \alpha}\right|^{2}\right)^{1 / 2}=\frac{1}{3} q \times \exp (-W)$; this expression is interesting from the point-of-view of mathematical physics since its left-hand side represents a norm associated with a certain Hilbert space equipped with the scalar product $\left\langle\overrightarrow{\mathrm{u}}_{\mathrm{n} \alpha}, \overrightarrow{\mathrm{w}}_{\mathrm{n} \alpha}\right\rangle=\sum_{\mathrm{n}, \alpha}^{\mathrm{N}}\left(\overrightarrow{\mathrm{u}}_{\mathrm{n} \alpha} \cdot \overrightarrow{\mathrm{w}}_{\mathrm{n} \alpha}\right)$, where the dot means usual scalar product. Moreover, we recall the physical sense of $S^{\prime}(\vec{q}, E)$; this quantity represents energy loss spectra.

Finally, we recall also the importance of eq. (11). The left-hand side of this is the structural-disorder contribution to the sum rule $\int_{\Omega} \omega \xi_{2}(\omega) \mathrm{d} \omega$.

\section{APPENDIX}

In fig. A1 we can compare the energy loss spectrum $S^{\prime}(\vec{q}, E)$ with $g(E)$ for $q=$ $17.0 \AA^{-1}$ and amorphous Ge, by using a periodic network model (see ref. [3]). In 


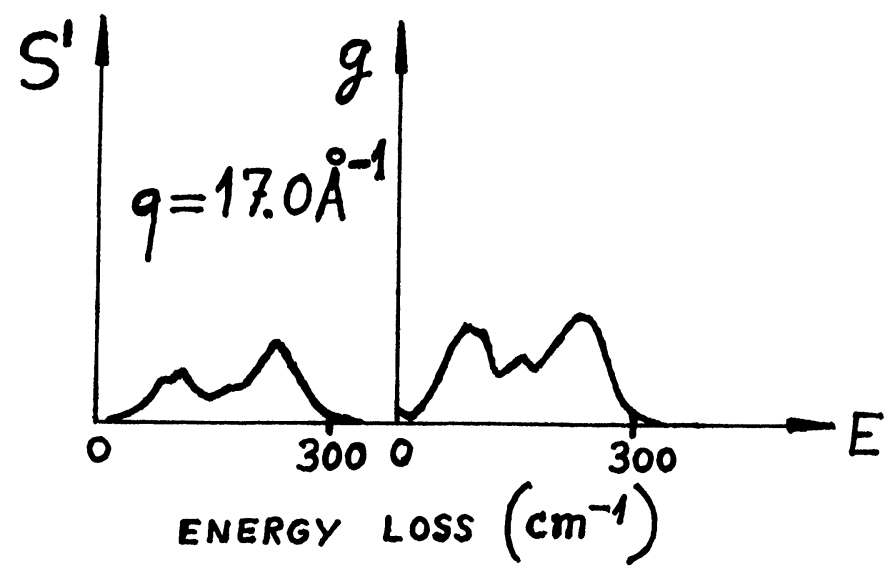

FIGURE A1 Plots of $\mathrm{S}^{\prime}$ and $\mathrm{g}$ for amorphous $\mathrm{Ge}$ with respect to a periodic network model.

addition, it is interesting to note the comparison between the plot of $\mathrm{g}(\mathrm{E})$ and eq. (4).

\section{REFERENCES}

1. G. Nilsson and G. Nelin, Phys. Rev. B 3 (1971), 364.

2. R.J. Temkin, W. Paul, and G.A.N. Connell, Advan. Phys. 22 (1973), 531.

3. R. Alben, AIP Conf. Proc. no. 20 (1974), 249-259.

4. W. Marshall and S.W. Lovesey; Thermal Neutron Scattering, (Oxford, 1971).

5. J.D. Axe, D.T. Keating, G.S. Cargill III, and R. Alben, AIP Conf. Proc. no. (20) (1974), 280.

6. I.B. Gel'fand and G.E. Shilov; Generalized Functions, vol. 1, (Academic Press, 1964).

7. I.B. Gel'fand and N. Ya. Vilenkin; Generalized Functions, vol. 4 (Academic Press, 1964).

8. S.S. Mitra, D.K. Paul, and Y.F. Tsay, AIP Conf. Proc. no. 20 (1974), 287.

9. S.S. Mitra, Y.F. Tsay, D.K. Paul, and B. Bendow, Phys. Rev. B 15 (1977), 4007.

10. M.A. Grado and M. Grado, Phys. Lett. A 169, no. 5 (1992), 400. 

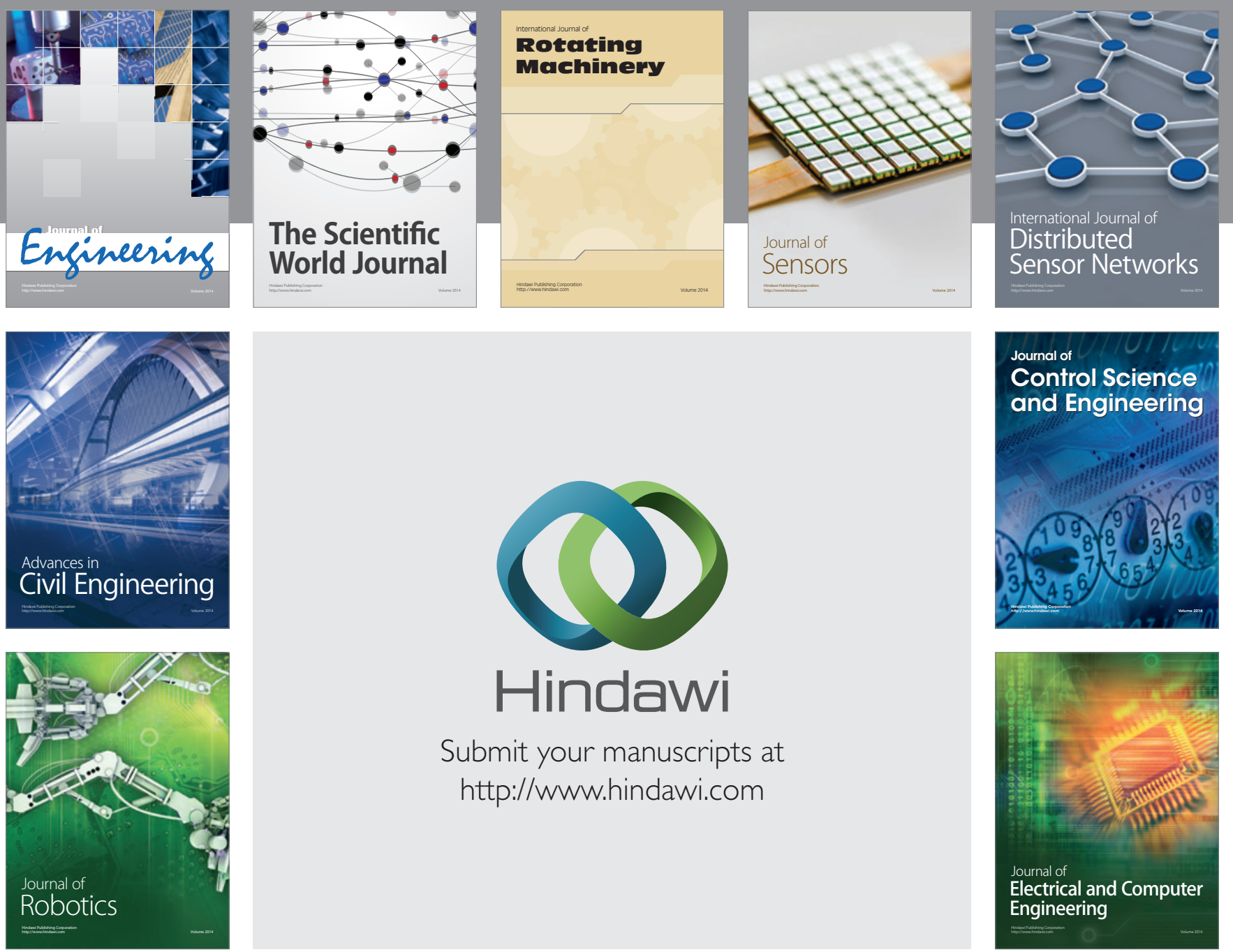

Submit your manuscripts at

http://www.hindawi.com
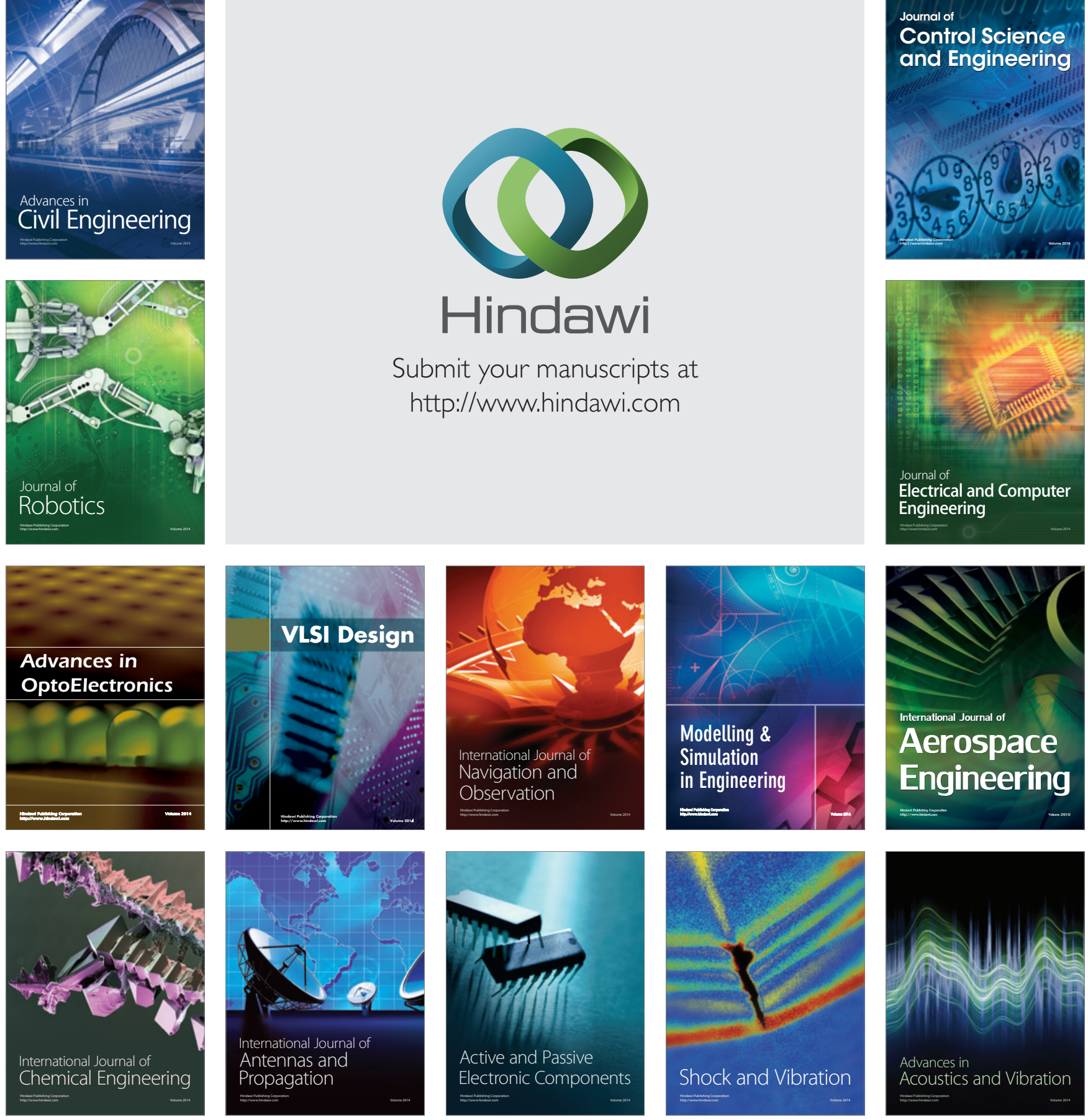